Review:

\title{
A Philosophical Inquiry into the Confusion over the Radiation Exposure Problem
}

\author{
Masaki Ichinose $^{\dagger}$ \\ The University of Tokyo \\ 7-3-1 Hongo, Bunkyo-ku, Tokyo 113-0033, Japan \\ †Corresponding author, E-mail: ichinose@1.u-tokyo.ac.jp \\ [Received December 24, 2015; accepted January 29, 2016]
}

\begin{abstract}
In this paper, I discuss from a philosophical viewpoint the so-called radiation problem that resulted from the Fukushima Daiichi Nuclear Power Station accident after the Great East Japan Earthquake in 2011. The starting point lies in the conceptual distinction between "damage due to radiation" and "damage caused by avoiding radiation." We can recognize the direct "damage due to radiation" in Fukushima as not serious based on the empirical data so that I focus upon the problem of the "damage caused by avoiding radiation," particularly the damage due to evacuation actions. Actually, evacuation actions caused more refugees to die of suicide and diseases than supposed. Obviously, there is a practical problem on whether the forceful and emergent evacuation was needed. In addition, I will point out that some people psychologically had negative feelings about the radiation problem altogether, for example, absurdity, discomfort, anxiety, or distrust, where some of them tend to twistedly solve those by giving moral censure to people and the product in affected areas. This brought about serious harm to people in Fukushima. I will interpret some people's careless adoption of precautionary principle and their misunderstanding of the legal standard in radiation protection as being latent in this tragedy.
\end{abstract}

Keywords: the Fukushima Daiichi Nuclear Power Station accident, radiation exposure, evacuation, precautionary principle, legal standard in radiation protection

\section{1. "Harm" or "Damage" in the Radiation Problem}

In this paper, the author, a philosophical researcher, discusses the so-called radiation problem that resulted from the Fukushima Daiichi Nuclear Power Station accident following the Great East Japan Earthquake and the direction that the discussion on this problem should take, organizing the points of the argument from a philosophical viewpoint. The basic impetus of this paper is to focus on the problem around the "effects of radiation exposure on health" apart from the "pros and cons of nuclear power plants." The theme "effects of radiation exposure on health" is significant irrespectively of the "pros and cons of nuclear power plants" because we are always exposed to radiation and considerably so during some medical treatments or other situations. In addition, various problems caused by the nuclear accident cannot be solved simply by deciding to decommission nuclear power plants. I would like to assert that, if one associates the "effects of radiation exposure on health" and related problems with the "pros and cons of nuclear power plants," a political theme, or ideology, such understanding is biased. Therefore, this paper does not consider the "pros and cons of nuclear power plants."

Why are radiation problems controversial? This is because of the potential "harm" of radiation exposure. Therefore, "harm" or "damage" should be discussed first and foremost with regard to the radiation problem. So then, what is "harm?" The "harm" of a tsunami disaster is obvious. Almost 20 thousand victims were killed and myriads of people have had to take shelter after losing their homes. This is the main damage of the disaster called "3.11." Further, what is the "harm" caused by radioactive diffusion and radiation from the nuclear accident? This question is challenging with regard to radiation problems. It is true that people living in Fukushima and East Japan suffered from more unnecessary radiation exposure than in normal conditions when radioactive materials were released because of the nuclear accident. However, it is difficult to logically state that the release of radioactive materials or more radiation exposure than normal is "harmful." This is because it cannot be regarded as "harmful" if a small amount of released radiation materials or unnecessary additional exposure does not adversely affect one's health ${ }^{1}$. Moreover, as mentioned above, radiation exposure and even additional exposure occurred before the nuclear accident. The world and space are filled with radiation, regardless of the amount. However, can the difficulties of evacuees owing to forced and voluntary evacuation, including stress, health deterioration, increased suicides, and increased deaths, be considered "damage" and "actual harm"? Although this view makes

\footnotetext{
1. Refer to the following: "living organisms that lived and evolved on the earth acquired capability to repair DNA damage at the cell level caused by radiation and active oxygen inhaled and respond to decrease error rates. As a result, more radiation is required to cause the effective number of DNA cut (the number of effective hits). Considering DNA repair and adoptive responses, biological dose as a standard for "low dose" can be greater than the above 0.2 mGy' (Doi et al., 2007, p. 65).
} 


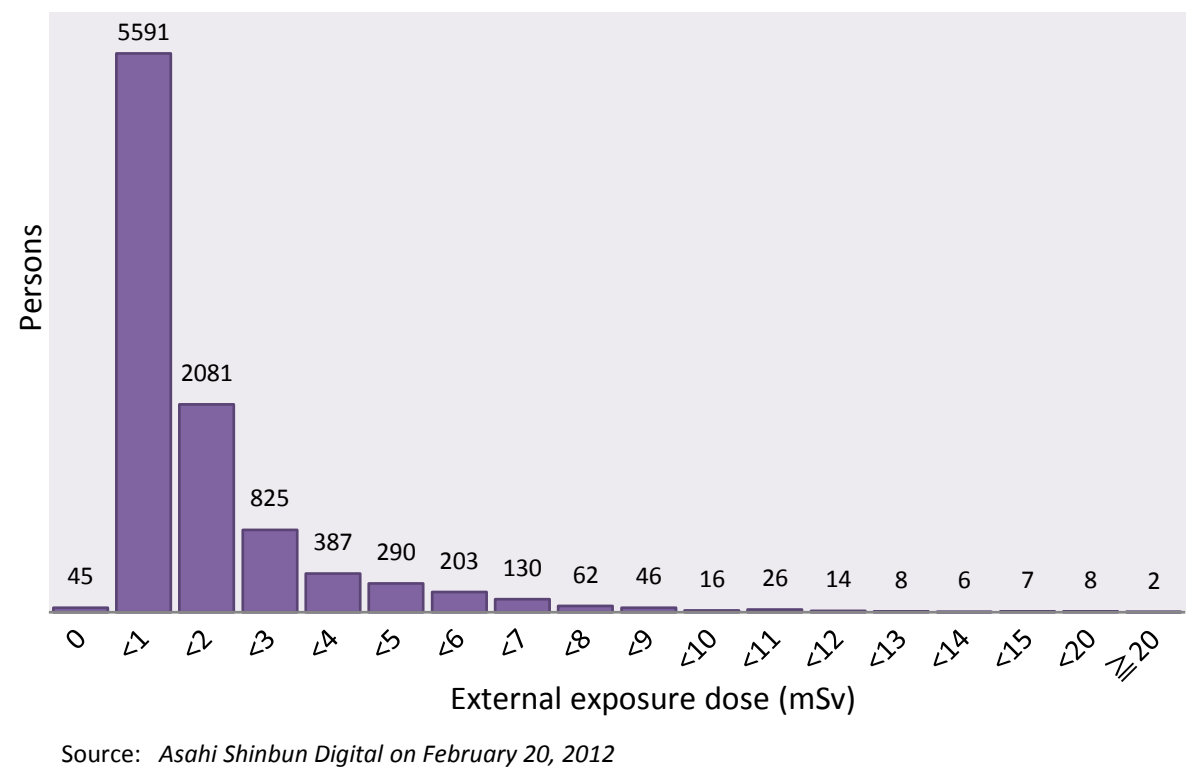

Fig. 1. Distribution of external exposure dose of Fukushima residents (mSv: millisieverts; excluding those involved in radiation work).

sense, the problem is whether the difficulties represent "damage due to radiation." Perhaps these effects should instead be called "damage caused by avoiding radiation."

Note that the concept of "damage" is used in a broad sense. In other words, "damage" here includes various matters that would not have occurred without the nuclear accident such as acute and late radiation disorder, deaths of disease and suicides, physical and mental health deterioration, anxiety and a sense of alienation, economic hardship, a pervasive sense of avoidance against produce from affected areas, education problems in children, domestic discord, and mental abuse (I personally suffered considerably from mental abuse that ignored the context in my argument by the skewed criticism of my peers). However, "deaths" as typical forms of "damage" are the main focus in this study.

\section{2. "Damage due to Radiation" and "Damage Caused by Avoiding Radiation"}

The conceptual differentiation between "damage due to radiation" and "damage caused by avoiding radiation" is the starting point for addressing the radiation problem. This differentiation can also be expressed as an introduction of the concept that it is rational to select options with less risk by differentiating and comparing the risks of radiation exposure versus that of evacuation to avoid radiation exposure. The risk of radiation exposure can roughly be calculated if the radiation dose is known. Regarding the Fukushima Daiichi Nuclear Power Station accident, additional and unnecessary external exposure was calculated to be within $5 \mathrm{mSv}$ in many cases of victims in Fukushima (mostly within $1 \mathrm{mSv}$ ), while internal exposure registers almost zero at presently, four years after the accident, even though the very small dose of estimated initial exposure was conjectured.

Let us look at the data. According to "Asahi Shinbun Digital," on February 20, 2012, the distribution of external exposure dose owing to the Fukushima Daiichi Nuclear Power Station accident, shown in Fig. 1, was obtained from 9750 residents in Namie Town, Iitate Village, and Kawamata Village of Fukushima, which excluded workers from the nuclear power plants, for the four months following the accident ${ }^{2}$.

Radiation doses for more than $94 \%$ of residents were at $5 \mathrm{mSv}$ or less, and those of almost $99 \%$ of residents were at $10 \mathrm{mSv}$ or less. As areas of the research matrix are close to the Power Station and suffered from relatively high doses of radiation, the doses of radiation were probably lower in other areas in Fukushima. The above data obtained are for four months; therefore, yearly doses would be higher. However, yearly doses would be much lower than that of the value multiplied by three because of natural attenuation (dilution owing to diffusion and settlement in the earth).

The website of Fukushima City includes measurement results of effective doses using glass badges for three months from September to November 2013 for 10,100 students of elementary and junior-high school, which indicated a significant decrease in radiation doses. Fig. 2 lists estimated values of yearly doses based on the measurements from these three months ${ }^{3}$. Its vertical axis stands for the number of people, and the horizontal one $\mathrm{mSv}$ per year.

More than $93 \%$ of subjects showed radiation doses of $1 \mathrm{mSv}$ or less per year, whereas $99 \%$ showed radiation doses of $2 \mathrm{mSv}$ or less. In some cases, external radiation

2. http://www.asahi.com/health/news/TKY201202200195.html 3. http://www.city.fukushima.jp/uploaded/attachment/29075.pdf 


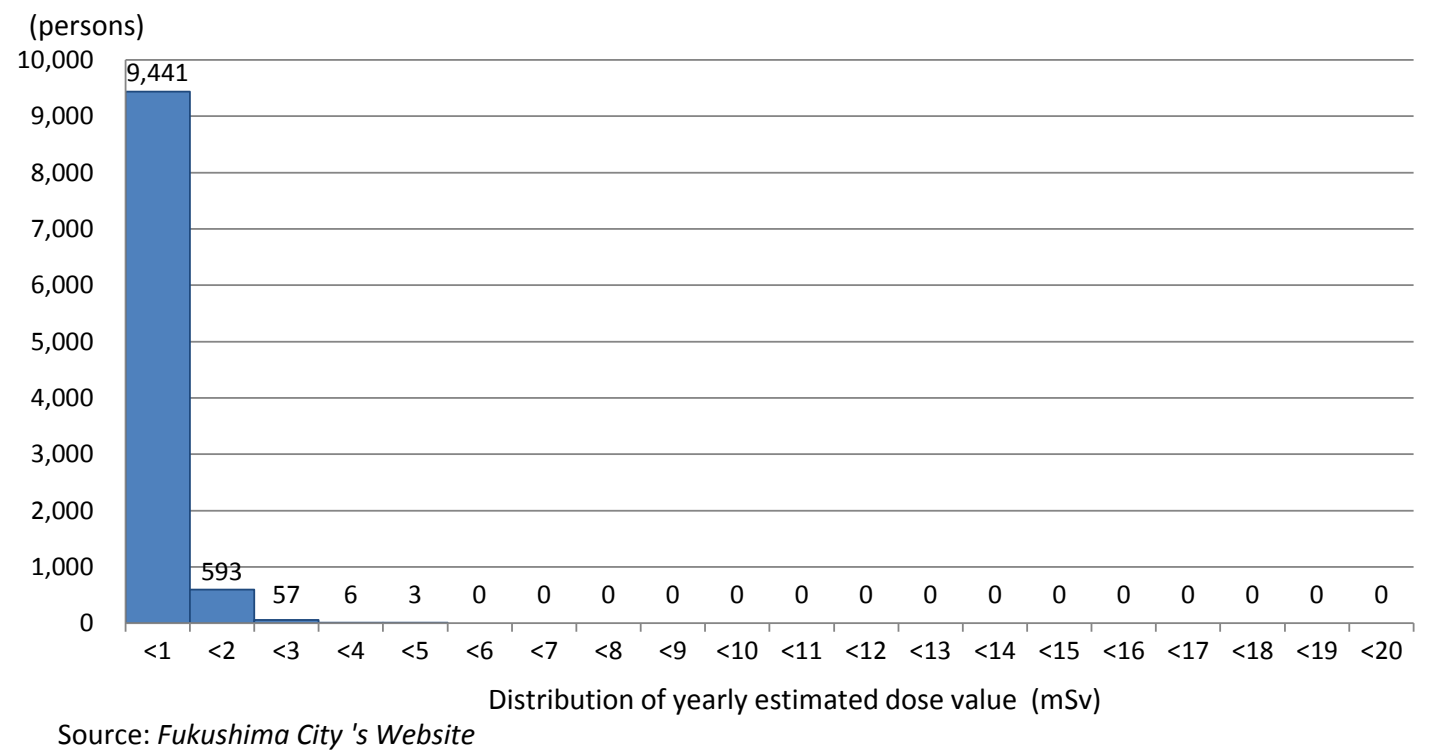

Fig. 2. Distribution of yearly estimated dose value measured by Fukushima City.

exposure is evaluated by multiplying the air dose per hour, which is obtained by monitoring posts, by 24 and 365; but the effective dose from external radiation is even lower than the values of the air dose multiplied by 24 and 365, as indicated by the above data obtained by a worn gauge for accumulated doses such as glass batches. An approximate comparison through the website of Fukushima University in Fukushima City reveals that the air dose on the "road in front of the center of dormitory" of the Kanayagawa campus on November 7, 2013 was $0.3 \mathrm{mSv}$ per hour ${ }^{4}$. When multiplied by 24 and 365, the value is $2628 \mu \mathrm{Sv}$, or $2.628 \mathrm{mSv}$. Compared to this, the accumulated dose obtained by glass batches above is significantly lower. In the formal criteria, the actual radiation dose is calculated by (air dose $\times 8+$ air dose $\times 16 \times 0.4$ ) assuming that one spends 16 hours indoors per day and that the effects of radiation are reduced by $40 \%$ indoors (See Tasaki 2012, p. 93). Since radiation is blocked by buildings or other objects, the actual radiation dose is estimated to be almost half of the value simply calculated using the air dose. In fact, measured values can be lower than estimated values.

In any case, radiation exposure risks should be evaluated by taking the values of effective doses based on accumulated dose gauges. Furthermore, radiation materials from the nuclear power plants will naturally attenuate owing to diffusion into the air and the ground before the physical half-lives. Alternatively, some materials are estimated to be stable and closely bonded to soil particles without being absorbed into plants or mixing with the water. Sho Shiozawa said "Cs is soluble in water to form cations and it is characterized by the fact that it is easily fixed to soil and organic substances such as living plants and does not readily move with water" (Shiozawa 2012, p. 15). Overall, risks of cancer deaths due to the Fukushima power plant accident are currently extremely low, exclud-

4. http://www.fukushima-uac.jp/guidance/top/fukudai-housyasen-H25 .html\#toku ing workers from the plants, even if the "LNT hypothesis" is adopted ${ }^{5}$. As for internal radiation exposure, detailed and accurate measurement results using whole body counters have been reported by Ryugo Hayano, a physicist, and Tsubokura Masaharu, a doctor. It was clarified that internal radiation exposure was almost 0 in April 2013, even though there had been initial radiation exposure by radioactive iodine in the first phase of the accident ${ }^{6}$. For

5. Kunikazu Noguchi remarked as follows on Date City, Fukushima Prefecture: "If appropriate dose reduction measure such as decontamination in living areas are taken for residents with higher external radiation exposure dose, evacuation is unnecessary. It is considered that external radiation dose is further reduced in the city more than one year after June 2013. Considering the situation in Date City, I think people can live in the northern and central areas of the prefecture at ease (Kodama, Shimizu, Noguchi 2014, p. 88). At the moment, risks to concern can be omitted.

6. Shinji Tokonami from the Hirosaki University reported investigation results on the front page of morning "Asahi Shimbun" on March 9, 2012, on initial exposure of radioactive iodine: Some people were exposed to almost $90 \mathrm{mSv}$ of radioactive iodine to the thyroid immediately after the nuclear power plant accident. However, this is the equivalent dose to thyroid and different from the effective dose used for radiation protection. Considering that almost all iodine gathers in the thyroid, an estimated value of initial exposure can be obtained by converting equivalent doses of thyroid to effective dose. Equivalent doses of thyroid can be transformed to effective doses by multiplying by 0.04 , the tissue-weighting coefficient. The resultant effective dose is $3.6 \mathrm{mSv}$. This is the maximum estimated value for initial exposure reported in "Asahi Shimbun." Immediately after the accident, the equivalent dose was confused with the effective dose, prompting turmoil.

The fact that the air dose was considered equivalent to the effective dose to the body was also a factor of confusion. Normally, the effective dose is one-fifth of the air dose. More specifically, according to Masaharu Tsubokura, who works in affected areas, 'air dose of $0.23 \mu \mathrm{v} / \mathrm{h}$ ' is the value obtained by calculating the "yearly additional $1 \mathrm{mSv}$ " per hour. The government has the target of $1 \mathrm{mSv}$ per year of additional exposure in the long run ... From the research results using Glass badges from September to November 2012, the results of 520 pupils who wore glass badges firmly were adopted. The values indicated by the glass badges are known to be very close to the damage amount (effective dose) to the body. The study compared the values of glass badges and exposure amounts that were estimated by the method designated by the government based on air doses in front of subjects' homes. Results were ...that exposure amounts estimated by the method designated by the government were approximately three times the values obtained using glass badges. In other words, when one lives in places with air doses of $0.6 \mu \mathrm{Sv} / \mathrm{h}$, they are exposed to radiation of $1 \mathrm{mSv}$ per year' (http//apital.asahi.com/article/fukushima/2012012 000016.html). Something was wrong when considering the dose. This experience should be transmitted to the world and next generations. 
example, they said "radioactive cesium was not detected from 'all' students of elementary and junior-high schools in Miharu town in medical examination in the fall 2013" (Hayano, Tsubokura, et al., 2013, p. 161). The question then is how to evaluate these radiation exposure dose values.

Such estimation is based not only on epidemiological evidence but also on pure logic. My reasoning is as follows: During medical treatments, we are sometimes exposed to doses of radiation greater than that of the Fukushima power plant accident; and if death risks due to the exposure dose in Fukushima is high, then our society would be a very short-lived one. Quite to the contrary, however, Japan is the world's top country for longevity. This reasonably suggests that radiation exposure at approximately $5 \mathrm{mSv}$ would not cause a significant increase in death risks ${ }^{7}$. Of course, radiation exposure risk is not 0 . However, such risks cannot be identified because they are minimal. Cancer death risks are complex, and multiple confounding factors should be considered. In this connection, although the epidemiological evidence is questioned and such questions make sense to some extent for cases of radiation exposure of $100 \mathrm{mSv}$ per year, they can be groundless for cases of radiation exposure of $5 \mathrm{mSv}$ or less per year. Thus, risks should be evaluated based on "quantities."

Moreover, even if these risks become a reality, they are late and cause only "possible" deaths. In contrast, "damages caused by avoiding radiation" including stress caused by evacuation actions, health deterioration, increased suicides, and increased deaths are not late but have occurred. The deaths of those who perished during evacuation are not "possible" but "actual" deaths. Clearly this point must not be neglected. From the perspective of lost life expectancy, the "damage caused by avoiding radiation" is far greater than "damage due to radiation" in the case of Fukushima.

\section{Damage due to Evacuation Actions}

The above notion can be understood by comparing "damage due to radiation" and "damage caused by avoiding evacuation" in the Fukushima power plant accident: namely, the comparison of health effects between (1) cases of possible "damage due to radiation" for those who remained in affected areas in Fukushima (except for those areas close to the Daiichi Nuclear Power Station) after the accident, and (2) cases of "damage by avoiding radiation" associated with evacuation. Regarding point (1), it was consoling that no one killed or seriously injured af-

7. According to the 2011 "New edition Life safety environment radiation" edited by the Nuclear Safety Research Association, the national dose of Japanese people is $5.98 \mathrm{mSv}$ per year, and the effective dose of natural radiation is $2.1 \mathrm{mSv}$ per year. Therefore, the average medical exposure of Japanese people is $3.88 \mathrm{mSv}$. Under this condition, the death rate is not significantly increased, rather longevity is promoted, so it would be safe to say that death risks would not considerably increased under exposure of approximately $5 \mathrm{mSv}$ per year (In most cases, those living in Fukushima are exposed to accumulated radiation of $1 \mathrm{mSv}$ or less per year). Refer to https://www.nsra.or.jp/library/book.html ter acute symptoms were reported. Therefore, late cancer and cancer deaths are the only problems of (1). In addition, stresses and anxieties when living in an environment with possible radiation exposure and the inconvenience of insufficient living conditions and infrastructure should also be considered.

According to the estimation as of 2012 made by Hoeve and Jacobson, environmental engineers from Stanford University, the number of lives saved by evacuation in the Fukushima power plant accident was three at the least, 28 as optimized, and 245 at most. Conversely, if (1) is selected and people remained in the affected areas, an additional 3 to 245 cancer deaths are estimated. In contrast, in the case of (2), or as evacuation actions were undertaken, more than 1800 victims died due to disaster-related causes. Disaster-related deaths in Fukushima Prefecture were far more than those in Miyagi Prefecture (approximately 900) and in Iwate Prefecture (approximately 400). The effects of the Fukushima power plant accident are obvious. More deaths were caused in the case of (2), when evacuation actions were taken (see Hoeve \& Jacobson, 2012). In addition, as discussed in the previous paragraph, no one was killed due to acute symptoms resulting from radiation; so the deaths of case (1) are estimated "possible" ones, while those of case (2) are "actual" deaths that have occurred. The difference in lost life expectancy can theoretically be regarded as infinite unless possible deaths in the future and deaths that have occurred are treated as equivalent from the standpoint of metaphysical fourdimensionalism.

I believe cancer deaths due to radiation exposure are a tragedy, without a doubt. At the same time, however, deaths due to evacuation activities are also surely tragic. I am firmly convinced that deaths due to evacuation efforts should also be considered to the same extent as cancer deaths due to radiation exposure. Some overly emphasize the dangers of radiation exposure, saying that "any kind of radiation exposure is dangerous" and "it is natural to feel anxiety about radiation exposure," and they are treated as if they embodied social justice in many cases. However, this absolutely does not mean deaths due to evacuation actions need not be considered. All "deaths" are sad. Moreover, "deaths" that are about to occur are more significant than "deaths" that might occur in the future. Discourses that do not deal with such significant "deaths" are dishonest and harmful to the society. Tragedy and damage such as the significant "deaths" that are about to occur must be prevented by the whole society.

Related to this point, after I published A philosophy confronting the problem of radiation exposure, researchers from the Faculty of Medicine, the University of Tokyo, where I am based, released research results of a "retrospective cohort analysis" called "Death risk analysis for elderly citizens due to evacuation after the Fukushima nuclear power plant accident." The report includes the first detailed data, which seem to be the most reliable presently available on evacuation-related deaths after the Fukushima nuclear power plant accident. The main findings of the report can be summarized as follows: 
- The death rate of elderly citizens increased 2.7 -fold after evacuation.

- However, the death rates differ depending on evacuation facilities, requiring consideration of evacuation processes, services, and nursing care and food in each facility.

- The risks of first evacuation are higher than those of successive evacuations.

- Evacuation of elderly citizens comprehensively related to deaths, and evacuation immediately after the accident was not necessarily the optimal selection.

- Rather than an "immediate evacuation," staying in familiar environments should be prioritized to wait and see about the circumstance; and care must be enhanced in shelters if evacuation is necessary.

- The risks of evacuation must also be considered in future disasters.

(Nomura, Gilmour, Tsubokura, Yoneoka, Sugimoto, Oikawa, Kami, and Shibuya 2013)

We should sincerely listen to such reports on actual damages and resultant proposals. While the report covered elderly citizens, it can be easily be conjectured that other citizens suffered from damages other than deaths. Evacuation includes different types ranging between forced and voluntary evacuations, and the differences in evacuations between those who moved to other areas in Fukushima Prefecture and those moved outside it are significant. In addition, family composition, household budget, and health conditions vary widely. However, a macroscopic viewpoint to roughly categorize objects is inevitable in statistical and epidemiological thought. There is no need to reject such human wisdom.

I have good reason to highlight the significance of classification of the forms of "evacuation." I think there are two objections that always arise when discussing the "damage" resulting from evacuation activities; these are potential factors to expand the range of actual damage as can be seen in the present case. The two objections are: (i) Does evacuation-related damage derive from the nuclear accident and radioactive materials diffusion, since it would not been caused without the nuclear accident? (ii) Are evacuees criticized if evacuation activities are generally regarded as harmful? As I mentioned, evacuation activities vary so widely that it is too rough to generalize their activities as "evacuation" as a whole. Concerning (i), I approve of the causal specificity using the counterfactual conditional sentence "evacuation-related deaths would not have occurred without the nuclear accident." However, the question is whether there were other factors that could have been artificially handled between the occurrence of the nuclear accident and the ensuing damage. I want to make it clear that the counterfactual conditional sentence "so many people would not have suffered damage if forced evacuation actions had not been taken," which uses a temporal axis extending beyond the nuclear accident, is worth considering (this point was studied in
Ichinose (2015a)). Staying rather than evacuating could be feasible even after the nuclear accident, as opposed to the fact that the nuclear accident had already happened and could not be canceled after the accident. Thus, the cause of the related damage could theoretically be attributed to the evacuation actions. More accurately, the possibility of such causal specificity should be considered at least in order to objectively understand the circumstances. By doing so, the generally accepted notion that radiation should definitely be avoided would be reflected upon in a calm manner; thus, current damage would be reduced and lessons for the future provided for certain.

Counterfactual conditional sentences with antecedents retrospective to pre-accident such as "if the nuclear power plant had been decommissioned" and "if facilities including backup power sources had been better prepared" can justly be connected to the consequent "many people would not have suffered damage," as well as counterfactual conditional sentences with antecedents focusing on a post-accident "if forced evacuation actions had not been taken." The decisive point here is the philosophical question regarding which counterfactual conditional sentence should be adopted as the most acceptable. Regarding this philosophical question, I hypothetically propose a policy to estimate the degree of acceptability of a counterfactual conditional sentence using a function of "manipulability" and "temporal distance" from the consequence. I posit that the most immediate way with the highest manipulability is the most realistic (not radical, at a low cost, and with the fewest side effects). When temporal factors are disregarded, the degrees of manipulability of decommissioning nuclear power plant, preparation of backup power sources, and cancellation of forced evacuation activities is not 0 . Let us suppose that the three degrees of manipulability are equal (which is reasonable enough as temporal factors were disregarded). However, even if we adopt this supposition, the temporal distance from the consequence (increased disaster-related deaths) is clearly the shortest for the cancellation of forced evacuation activities. According to this idea, the counterfactual conditional sentence "if forced evacuation activities had not been taken, so many people would not have suffered damage" is the most realistic and acceptable.

Some may argue that my discussion compels people to tolerate radiation exposure, but this argument is a straw man. The key problem is ultimately the health problems due to the nuclear accident, and thus the "life" problem. Therefore, health and "lives" are prioritized. It would do more harm than good if health deteriorates and "lives" are threatened by avoiding radiation exposure. I am not insisting on tolerance to radiation exposure, but am proposing to protect health and "lives" by doing our best in actual circumstances. We must not lose sight of our true goal.

I will sincerely respond to objection (ii) above. Evacuation activities immediately after the accident when there was no sufficient information do not deserve any indictment. I would have undertaken evacuation activities likewise if I had lived near the Fukushima Daiichi Nuclear Power Station. I suggest considering the option of going 
home if one finds difficulties in the evacuation activities after a certain period of time and if their home is safe to live in when the quantity of radiation material diffusion due to the accident is largely identified. I admit that some victims may choose to live in a shelter and this choice may be better in terms of risk management in many cases. Yet, some might oppose to me by saying that if my assertion has only such a narrow implication, it is too rough to classify 'damage due to evacuation activities' as one type. I can respond to this opposition, 'The rough classification is effective when grasping epidemiologic phenomena from the macroscopic viewpoint; and the possible causal relationship (or correlation relationship at least) obtained using this classification will certainly facilitate policy-making under similar circumstances in the future.' On the contrary, rejecting such an understanding because of a wide variety of circumstances will lead to the risk of increasing actual damages by repeating the same mistakes in the future: The possibility of staying in their home is concealed, and avoiding radiation exposure is retained as the only option.

Someone may counter that evacuation deaths should be prevented by improving evacuation facilities rather than staying home even if they admitted the circumstances surrounding disaster-related damage. This is based on the view that because radiation exposure is dangerous, evacuation is the first priority and damages due to evacuation should be avoided by preparedness. However, this view includes two issues: 1) The fact that quantity matters in radiation exposure is neglected. If the quantity or the dose is small, then people do not have to rush to shelters and leave their homes. The situation should be reviewed before making a decision. 2) The possibility of taking adequate measures to enhance evacuation facilities in advance should be questioned. If the same level of livelihood can be maintained after evacuation or movement (in terms of physical, mental, economic, and social aspects), then no evacuation-related deaths will occur. However, is it possible to realize such ideal preparedness? I think that would be very difficult because of physical and financial problems. The manipulability of such preparedness is quite low. In fact, no states and societies have been able to afford to do so. Consequently, it should be clearly noted that evacuation has death risks including a risk in the sense of the difficulty of enhancing evacuation facilities beforehand when addressing problems in future disasters. People are presumably aware of this point using some imagination. Imagine what you might feel if you were instructed to immediately take in refugees for about one year. The difficulty will quickly be understood.

\section{4. "Sensation of "Not"' and "Moral Dilemma"}

However, why did the above, easy-to-understand position not prevail? Why was difficult evacuation, or more precisely, only difficult evacuation recommended? More precisely, why were so many people firmly convinced that they had no option but to evacuate under pressure, and why had they to die? By addressing these questions, the real nature of damage of the radiation problem due to the Fukushima nuclear power plant accident is highlighted. I think what I want to call the sensation of "not" made the radiation problem more confusing - this sensation includes a "sense of the absurd" and a "sense of discomfort" resulting from unnecessary, useless, additional radiation exposure as a fault of the government and power company, a "sense of anxiety" about future health conditions, and a "sense of distrust" for information transmission from the relevant agencies that were sometimes incorrect and changing. The real nature of the damage of the radiation problem and the diffusion of radiation materials seems to originate from the sensation of "not" in a way.

The background of the sensation of "not" is that we regard radiation as a threat, or an icon of fear. Japanese people especially feel this way because we experienced Hiroshima, Nagasaki, the Daigo Fukuryu Maru, and the JCO critical accident. Furthermore, the radiation problem can be deemed a man-made disaster, unlike tsunami disasters. When responsibility is accountable (and it should be strictly pursued) and anger can be directed to some entities, one's sense of the absurd, anxiety, and discomfort increases. It should also be taken into account that the understanding of general citizens who have silently accepted nuclear power plant administration as being partly responsible for the nuclear accident, in turn becomes a selfdeceiving sense of distrust and discomfort. The sensation of "not" manifests as an unconscious, self-defensive response to disguise one's own errors.

When the sensation of "not" becomes strong enough, one will avoid the sensation. The sense of the absurd and discomfort are associated with anger and movements toward investigation of responsibility, leading to movements against the nuclear power plant. The society is then sunk in a morass; a stressful world appears in which prospects for the future are hard to attain. In addition, the sense of anxiety emerged as immediate evacuation, worsening the situation in some cases. The sense of distrust produced an unproductive reality where experts on radiation protection and radiation medicine were accused of being "scholars patronized by the government" in addition to the general condemnation against the government and power company. In such a way, the sensation of "not" turned to a negative feeling as a matter of course.

On top of these negative circumstances, a more serious one has arisen: many people take actions to avoid the sensation of "not," but this action in turn does harm to some people. Movements against nuclear power plants are decisively persuasive because a nuclear power plant accident can endanger human lives. Recall the JCO critical accident in 1999. However, to decommision nuclear power plants altogether takes time; the problem of the time required for decommissioning a reactor and the issue of waste disposal are undeniable. Moreover, these problems cannot be solved by a single country. Nuclear power plants in neighboring and other countries relate to us and to all of humanity. So then, movements toward 
the decommissioning of nuclear power plants should expect a continual struggle requiring considerable endeavor. However, as described above, those in affected areas will still feel anxieties confronting the danger of death at this moment. Currently, urgent demands are for the provision of medical services and increases in administrative health nurses caring for individuals frequently. From the perspective of the victims, movements against nuclear power plants in Tokyo or other areas are somewhat unsympathetic. The sense of danger for nuclear power plants can be shared (although I understand the danger of thermal power plants in multi-layered meanings as well). However, the question remains of how to deal with the ongoing problems in affected areas. Might direct supports for those who are trying to restore their lives and tolerate radiation exposure be possible? Can the energy for demonstrations against nuclear power plants be directed instead to direct supports for affected people? Is the order of priorities incorrect? It is inevitable to yield to such a sense of frustration.

In other words, the responses to a sense of the absurd and to discomfort produce a harmful sense of alienation, or being-left-alone for affected people although those responses are based on a morally justifiable motivation (respect for human lives). With regard to this point, I argued in another paper that the "metaphysical approach," which addresses extensive, long-term issues such as the pros and cons of nuclear power plants, and the "epistemological approach," which intends to solve urgent, actual damage in the present, are different impulses and sometimes compete with each other when confronting the nuclear accident. The two approaches should not be confused with each other. My interpretation is that such confusion is one factor in the disarray related to the radiation problem (refer to Ichinose, 2015a, p. 25).

Furthermore, a large number of people who lived in East Japan including Fukushima Prefecture took refuge driven by anxiety about future health, especially for their children, which caused harm. I mentioned the fact that evacuation increased deaths. In addition to this, evacuation activities inversely caused harm to those who remained in the affected area through moral censure to the decision to remain in evacuation recommendation areas (children may be endangered), discrimination against those remaining in evacuation recommendation areas, and a pervasive sense of avoidance to produce from evacuation recommendation areas. The scheme in which actions based on a morally justifiable motivation to protect children's health do harm to those in affected areas is also apparent here ${ }^{8}$. It is difficult to make assertive remarks about whether to evacuate or not based on current radiation doses in Fukushima. One can evacuate if they want and the radiation exposure dose will be reduced by evacu-

8. Proposals for temporal refuge for affected children imply that it is dangerous to stay in affected areas, doing harm to those still living in affected areas, although they are from good intention that can morally be justified. Judgment must be based on "dose" and individual decision-making must be respected. Careless proposals neglecting data from outside affected areas are not allowed even if they are from good intentions. The aphorism "the road to hell is paved with good intentions" should be recalled. ating; this does not mean that staying in affected areas will surely be hazardous to one's life. Rather, it would be safe to say that staying in affected areas will not jeopardize one's life. Radiation doses are so low that one can live in affected areas with appropriate attention. The action policy is judged by each individual. Based on this background, I want to focus on the tragedy in which morally justifiable evacuation actions do harm to others.

Similarly, experts were rejected by some people as "scholars patronized by the government" because of their sense of distrust. This rejection or abuse may originate in a sense of justice. However, by doing so, researchers who try to make sincere statements based on their expertise and experience may be compromised or hesitate to make statements, resulting in confusion and depriving affected people of opportunities to access the opinions of experts. Here again, actions from morally acceptable motivations do harm to others. I call this situation a "moral dilemma." I would like to express my opinion that the core or the nature of the present radiation problem is a "moral dilemma" emerging from the sensation of "not."

\section{Causal Relationship Between Radiation Ex- posure and Cancer Deaths}

How can we get out of this "moral dilemma?" It is my understanding that a possible course is to correctly and objectively estimate the health effects of additional radiation exposure caused by the nuclear accident on the human body using the presently available collective knowledge of human beings. In other words, the source of the sensation of "not," especially the "sense of anxiety" needs be examined in detail as to whether it is a truly justified sense or not, and we should make efforts to patiently remove the "sense of anxiety" when it is likely to be unnecessary. Never evaluate the sense of anxiety of people as "natural anxiety" from the outset. This is begging the question, and it causes a hundred harms and not a single gain because confusion and anxiety will only increase. In fact, the most sincere and effective way is to calmly consider "degrees" of danger and to repeat examinations gradually, although this method is seemingly long.

To help follow this plan, I want to propose a question on the causal relationship between radiation exposure and cancer deaths from the perspective of a philosophy researcher, as partly described above in relation to evacuation-related deaths. To estimate the health effects of radiation "correctly and objectively" is nothing less than to calmly discern the "causal relationship" between radiation exposure and its effects on the human body. Firstly, an obvious point from the beginning is as follows: The causal relationship in question is NOT the "necessary" causal relationship without exception. The problem is the causal relationship between radiation exposure and increased cancer death rates, provisionary setting aside the issue of the exposure amount. It is clear that "probability" matters here. Such a causal relationship is called "probabilistic causality" in the philosophical context. The 
simplest form of probabilistic causality can be expressed using the inequality relationship of "conditional probabilities" (hereafter referred to as "Equation P") as follows:

$$
\text { Equation } \mathrm{P}: \mathrm{P}(\mathrm{E} \mid \mathrm{C})>\mathrm{P}(\mathrm{E} \mid \sim \mathrm{C})
$$

The idea is that, when comparing "the probability of $\mathrm{E}$ when C occurs" and "the probability of E when C does not occur," the former is larger; then, for the time being, "C is the cause of E" can be regarded as true. To apply this to the present case, "radiation exposure of general victims due to the Fukushima nuclear power plant accident (excluding workers in the plant)" is substituted with " $C$," and those who "die of cancer" with "E." If Equation P holds true, then the causal relationship between radiation exposure and cancer deaths can temporarily be expected.

However, we have theoretical issues piling up. The question of whether the inequality relationship of Equation $\mathrm{P}$ holds true arises in the case of Fukushima when considering the fact that the external radiation exposure was $5 \mathrm{mSv}$ or less and the internal exposure was almost 0 , combined with the findings regarding the radiation exposure of human beings thus far. This point is highly doubtful from a logical point of view; as mentioned above, we are sometimes exposed to radiation doses equivalent to that of the power plant accident during medical treatments; nevertheless, we do not live shorter, but longer. We are sometimes exposed to additional radiation: natural radiation from soil (recall Kerala in India and Ramsar in Iran), cosmic radiation when boarding airplanes, external and internal exposure in radon spring, and exposure from granite buildings (e.g. the Grand Central Station in N.Y. and the Diet Building in Japan). If accumulated, exposure doses may be above $5 \mathrm{mSv}$. However, such radiation exposure is not controversial in the world except for cases of excessive medical exposure. To be precise, as long as a specific phenomenon has not occurred, such exposure is not discussed. If that is the case, it can be reasoned that demonstrating the relationship in Equation $\mathrm{P}$ using data for the present levels of radiation exposure from the Fukushima nuclear power plant would be extremely difficult as long as the laws of the world are constant to some degree (this is our basic understanding of the world, although philosophers can question this).

Another emerging problem is the possibility of "common cause." Even if a clear relationship between radiation exposure due to the nuclear accident and cancer deaths is found applicable to Equation $\mathrm{P}$, the causal relationship between them cannot be determined immediately for theoretical reasons. For example, the possibility of the following case cannot be excluded: A "specific genetic condition" is a factor of increasing the possibility of "cancer deaths," and at the same time, the same genetic factor is the cause of inducing "damaged cells due to ionization and excitation resulting from radiation exposure." If such a common cause generates both results of "damaged cells due to ionization and excitation resulting from radiation exposure" and "cancer deaths," then cancer death rates remain the same even when radiation exposure is avoided.

In addition, some difficulties that statistic, epidemio- logic thoughts always encounter are anticipated such as "overdetermination" and "Simpson's paradox." "Overdetermination" means multiple factors cooperatively cause a single result; i.e., in the case of "cancer deaths," it is difficult to estimate the degrees of contribution of radiation exposure to the result because multiple factors such as diet habit, human relationships, smoking, sleeping, exercise are comprehensively entangled in addition to radiation exposure. This can be further explored from the philosophical viewpoint to find a fundamental difficulty in the individualization of phenomena: Which factor should be considered? The renowned "Simpson's paradox" is not detailed in this paper. In short, this is a traditional statistical paradox: In a statistical aggregation divided into Group A and Group B, even if a relationship between the same factors shows the same inequality relationship of Equation $\mathrm{P}$, the inequality relationship can be reversed when Group A and Group B are combined. It is difficult to completely delete the possibility of "Simpson's paradox" because a statistical survey necessitates a divided aggregation as the whole data cannot be obtained at once.

The above matters shed light on the fact that it is quite difficult theoretically to estimate the causal relationship between "radiation exposure" and "cancer deaths" in relation to the Fukushima nuclear power plant accident. This difficulty facilitates the generation of "moral dilemmas" in a sense. However, in spite of those difficulties, data for Equation $\mathrm{P}$ should be dealt with seriously, if available. Even though "probabilistic causality" contains the above difficulty in principal, there is no other way to support the causal relationship but to collect statistical and probability data. As far as I understand, causal relationships are established as "scenarios" that people postulate based on collected objective data. It is desirable to establish "scenarios" of more persuasive and reasonable causal relationships through social discussions by collecting objective data.

\section{Legal Standard and Precautionary Princi- ples}

However, it seems that these discussions are seldom accepted. The background of this is, I believe, the problem of the legal standard of " $1 \mathrm{mSv}$ per year" and the idea of "precautionary principles." These problems have brought about further confusion about the "moral dilemma."

Let us review the problem of the legal standard of " $1 \mathrm{mSv}$ per year." Immediately after the nuclear power plant accident, it was pointed out that " $1 \mathrm{mSv}$ per year" was stipulated in acts as the upper limit of the radiation exposure of the general public excluding natural and medical exposure; however, the value was exceeded due to the accident, violating the relevant acts. From then on, the standard of " $1 \mathrm{mSv}$ per year" has seemingly become a premise for discussion and decontamination work has been planned based on that value. However, the standard of " $1 \mathrm{mSv}$ per year" is not for the limitation of exposure for the general public but for released radiation doses in facilities handling radioactive materials in a precise sense. 
As a matter of fact, however, if the upper limit of released radiation dose in facilities handling radioactive materials is " $1 \mathrm{mSv}$ per year," then the upper limit of radiation doses for the general public must be " $1 \mathrm{mSv}$ per year" or lower. In addition, discussions have already taken place with presupposing that the radiation limit of the general public is " $1 \mathrm{mSv}$ per year." Under this scenario, the notion that "1 mSv per year" as the exposure limit should be temporarily accepted in order to ensure the possibility of general discussion, whereupon we need to examine the significance of the notion, even though we have to note that this exposure limit is not implying the real boundary to harm human bodies but is stipulated only by way of caution. In any case, if the upper limit of " $1 \mathrm{mSv}$ per year" for the general public were flatly rejected because there are no acts that support the value, then mutual communication would stagnate.

I felt strange about the use of " $1 \mathrm{mSv}$ per year" immediately after the accident because the value was wielded as an absolute authority like a royal coat of arms. However, a short inquiry can make us understand that the ground of the authority lies in this proposition:

'The legal standard of " $1 \mathrm{mSv}$ per year" ought to be observed.'

The absolute correctness of this proposition formed the core of the authority. However, the proposition is absolutely correct in the range it denotes, but the correctness of it is based on the definition of "law" concept: 'a law ought to be observed.' This can be expressed, as the proposition is a priori correct from the definition rather than from its meaning. In other words, the proposition proposes nothing with substance. It has only empty truth. However, if the correctness is set forth, and the absolute correctness is converted to authority, then this correctness is implicitly replaced with the a priori correctness of another proposition related to laws: 'criminal laws banning murder and violence ought to be observed.' Further, the correctness is replaced with the a priori correct proposition that the 'violation of criminal laws banning murder and violence is dangerous for the human body' by the definition of "murder and violence." As a result, the violation of the legal standard " $1 \mathrm{mSv}$ per year" is impressed as if it is dangerous for the human body. This is to say, "double replacement of a priori correctness." Needless to say, this is an unfair discussion. Without becoming obsessed with such a discussion, the relationship between radiation exposure and cancer deaths should be considered based on factual investigations.

In parallel with the discourse of " $1 \mathrm{mSv}$ per year," the fact that people have unconditionally accepted the application of the concept of "precautionary principle" to the radiation exposure problem induced the "moral dilemmas," preventing an appropriate causal relationship "scenario" based on objective data from being proposed. The "precautionary principle," roughly speaking, is the concept that if a specific activity or phenomena can possibly cause serious, irreversible damage, precautions to avoid the damage must be taken even when the damage is not sure. Many people insisted that the "precautionary principle" be applied to radiation exposure based on the intuition about dangerousness of radiation exposure, and this idea was disseminated. There are "weak" and "strong" versions of "precautionary principle," and their consistency is sometimes questioned. The "precautionary principle" was originally a decision-making principle in the context of environmental problems in Europe as opposed to those that consider probability and costs such as "costbenefit analysis." However, when one risk is avoided, another risk may be introduced. A typical example is when tap water was avoided because the chlorine in it is considered harmful to human body, but infectious diseases may still be caused if the chlorine is omitted. The following critical points have long been proposed: the "trade-off" between the "target risk" to be prevented and the "countervailing risk" caused by precautionary measures should be considered. This point was disregarded in the application of "precautionary principle" to the radiation exposure problem for some reason". In this case, the "target risk" is radiation exposure. What is the "countervailing risk?" The answer has been mentioned: health problems, increased deaths, discrimination of evacuees, avoidance of produce from affected areas, and stress caused by evacuation activities. We should take steps sincerely toward investigations of the causal relationship between radiation exposure and cancer deaths as well as the creation of a reasonable "scenario," without rushing to the quick and harmful response: any radiation exposure should be avoided (always remembering that "quantity" matters in the radiation problem).
Kyo Kageura asserted the applications of "precautionary principles" to about possible risks," and stated that the application is an "internationally shared understanding." (Ichinose et al., 2012. pp. 141-143). This notion is clearly in error, although it appeals to emotion and is accepted by people. With regard to "precautionary principles," because various international criticisms have been proposed including "countervailing risk," as discussed in the main body, "internationally shared understanding" is a factual error. Some states in the U.S. rejected applying "precautionary principles" to the danger of electromagnetic waves (Shimegi 2003, tity" that is fundamental in the radiation exposure problem, so it is an error and is harmful. As emphasized many times, if quantity is low, radiation exposure does not cause adverse health effects. To advocate an anxiety-provoking thing without this basic recognition urges people to have anxieties and promotes damage. I would urge those who have made such statements to reflect on the serious damage they have caused.

It is also noted that what "serious and irreversible" danger and damage are is also a problem related to "precautionary principles" in addition to "risk trade-off." Is "my death" included in such damage regardless of the "including staying up late)? All things are irreversible according to the "second law of thermodynamics." Via such discussions, a decisive counterargument is derived in relation to "precautionary principles." If "precautionary principles" are literally accepted, breathing and having food are prohibited because the world is filled with risks regardless of the amount. However, such the prohibition relates to "life." Thus, following "precautionary principles" causes serious and irreversible damage. As Sunstein pointed out, application of "precautionary principles" will result in "paralyzing" in which the application of "precautionary principles" itself is prohibited (Sunstein 2007, pp. 125-126). Some cases discuss the application of the precautionary principle with considering the magnitude of risks and the probability of the relevant phenomenon pecause they do not match the origin of "precautionary principles" that was proposed as an alternative of "cost-benefit analysis," or "risk trade-off analysis." 


\section{Survive with "Moral Dilemmas"}

We reach the conclusion. Radioactive materials were diffused due to the nuclear accident. We suffered from some additional radiation exposure. However, this does not inevitably do "harm" to us. Rather, it is reasonable to infer that the possibility of serious damage to human body is quite low based on the wisdom of human beings and the information from the Fukushima nuclear power plant. Yet, the anxieties of citizens cannot be easily dispelled. The motivation of voluntary evacuation can be morally justified and understood considering children's health, and thus the liberty of those who voluntarily evacuated should absolutely be respected. However, as discussed in relation to the "precautionary principle," an action for avoiding one risk may induce another risk, causing harm. Evacuation lives may impair the health of evacuees and evacuation actions can unintentionally highlight the fact that an affected area is too dangerous to live in, resulting in the avoidance of produce from affected areas. An action includes good and bad aspects. In this case, one option for individuals is to judge and make decisions by themselves, and to just practice without appealing to and recommending something to others. This is to adhere to one's own action policy without involving others and accusing others from their own perspective.

However, I estimate a more reasonable policy that minimizes damage as a whole: Except for areas close to the Fukushima Daiichi Nuclear Power Station, evacuees can go home if possible (unless their home is too damaged to inhabit because of their long absence) and return to their normal lives with reasonable care (to not continue eating home-grown vegetables or move close to hotspots unnecessarily), recognizing that serious damage is unlikely to occur in the current radiation exposure situation. This means to calmly receive objective data and make decisions related to one's life based on an understanding of rational, causal relationships. However, "more reasonable" is different from "surely reasonable." Those who want to evacuate and stay in shelters may be reasonable. One should have a strong mind if they want to reconstruct their lives on land with flexible container bags holding contaminated soil. I believe the way to survive with the "moral dilemmas" of the present radiation exposure problem is to practice and make decisions silently without involving others because each thing has good and bad aspects.

In any case, the health problem is to consider "life and death" in reverse. The disaster that occurred is regarded as a chance to consider "life and death" more deeply in order to obtain lessens for the future, keeping in mind that this will be linked to the eulogy for victims of the tsunami disaster.

\section{References:}

[1] R. Hayano, M. Tsubokura, M. Miyazaki, H. Satou, K. Satou, S. Masaki, and Y. Sakuma, "Internal radiocesium contamination of adults and children in Fukushima 7 to 20 months after the Fukushima NPP accident as measured by extensive whole-bodycounter surveys," Proceedings of the Japan Academy Series B, Physical and Biological Sciences, Vol.89, pp. 157-163, 2013.
[2] T. Hoeve and M. Z. Jacobson, "Worldwide health effects of the Fukushima Daiichi nuclear accident," Energy \& Environmental Science, DOI: 10.1039/c2ee22019a, 2012.

[3] M. Ichinose, "A philosophy confronting the problem of radiation exposure," Chikuma Publishing Company, 2013 (in Japanese).

[4] M. Ichinose, "Life is valuable, and life is mortal: Philosophical reconsideration on self-deception lurking within the debate concerning radiation exposure in the case of Fukushima," Philosophical Studies, Vol.33, pp. 1-48, The department of Philosophy, The University of Tokyo, 2015a (in Japanese).

[5] M. Ichinose, "A Philosophical fragment on "Life is mortal": Difference and Continuity between Humans and Animals," 'Inochi (Life)' Reconsidered, The Society of Philosophy, Japan, pp. 46-74, 2015b (in Japanese).

[6] M. Ichinose, K. Ito, K. Kageura, T. Kodama, S. Shimazono, and K. Nakagawa, "Morals on low dose radiation exposure," Kawade Shobo New Publishing Company, 2012 (in Japanese).

[7] K. Kodama, S. Shimizu, and K. Noguchi, "Science and social study of radiation exposure," Kamogawa publishing company, 2014 (in Japanese).

[8] S. Shiozawa, "Radiation contamination in rice fields and its transmission to rices," The Journal of the Japanese Society of Irrigation, Drainage, and Rural Engineering, Vol.89, No.7, pp. 15-18, 2012 (in Japanese).

[9] N. Shimegi, "Present Status of the Precautionary Principle on Risk Management," Bulletin of Seigakukan University, Vol.15, No.2, pp. 91-107, 2003 (in Japanese).

[10] S. Nomura, S. Gilmour, M. Tsubokura, D. Yoneoka, A. Sugimoto, T. Oikawa, M. Kami, and K. Shibuya, "Mortality Risk amongst Nursing Home Residents Evacuated after the Fukushima Nuclear Accident: A Retrospective Cohort Study," PLOS ONE on line, March 27, 2013.

[11] C. R. Sunstein, "Worst-Case Scenarios," Harvard University Press, 2007.

[12] H. Tasaki, "Elementary knowledge to live confronting troublesome radiation," Asahi Publishing Company, 2012 (in Japanese).

[13] M. Doi, R. Kanda, H. Yonehara, S. Yoshinaga, and Y. Shimada, "Low dose radiation and health effects," National Institute of Radiological Sciences, 2007 (in Japanese).

Name:
Masaki Ichinose
Affiliation:
Professor of Philosophy, Ph.D., The University
of Tokyo

